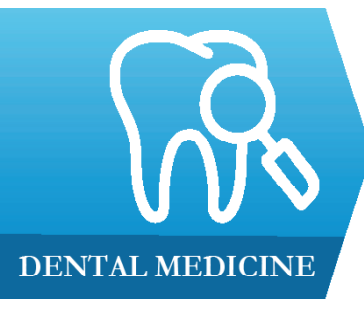

1) Department of Oral Radiology, Faculty of Dentistry, Iuliu Hatieganu University of Medicine and Pharmacy, Cluj-Napoca, Romania

2) Department of Maxillofacial Surgery, Faculty of Dentistry, Iuliu Hatieganu University of Medicine and Pharmacy, Cluj-Napoca, Romania

3) Department of Surgery (Dental and Maxillo-Facial Surgery), Faculty of Dentistry, Grigore T Popa University of Medicine and Pharmacy, Iasi, Romania

4) Department of Dental Medicine, University of Oradea, Romania

5) FSEGA, "Babes-Bolyai“" University, Cluj-Napoca, Romania

\title{
Financial efficiency estimation for a dental radiology laboratory
}

Maria Marcu ${ }^{1}$, Mihaela Hedesiu ${ }^{1}$, Loredana Bogdan ${ }^{1}$, Gabriel Armencea ${ }^{2}$, Avram Manea ${ }^{2}$, Raluca Roman ${ }^{1}$, Sergiu Vacaras ${ }^{2}$, Danisia $\mathrm{Haba}^{3}$, Anca Porumb ${ }^{4}$, Mihaela Baciut ${ }^{2}$, Sorana Crisan ${ }^{5}$

\begin{abstract}
Background and aims. Considering nowadays trend among dentists to install a radiology laboratory beside their current practice, we proposed to investigate the aspect of financial efficiency related to such investment.

Methods. We evaluate two existing options: simple investment, consisting of intra-oral equipment and accessories, or investment in a radiology center that includes panoramic and $\mathrm{CBCT}$ equipment. The initial investment includes equipment acquisition, fitting out of the location, radiology accreditation and other miscellaneous expenses. Costs were estimated based on current quotations on the specific market available in Romania. We also described a financial model to estimate the financial risk.

Results. The analysis was made under the assumptions that the laboratory is operated by the dentist who made the investment in the form of a legal person and paying corporate tax like all Romanian entities. The analysis took into account current fees for different types of X-rays, usual expenses of such a laboratory, and describes the approach to this analysis, starting with the initial investment estimation and forecast of revenues and expenses. Based on these projections and assessment of the working capital, we have built the cash flows forecast. Following a risk analysis we could assess the financial efficiency of the two investment alternatives.
\end{abstract}

Conclusions. Our study reveals that the radiology center represents a more profitable investment due to the higher economic return rate.

Keywords: finances, financial risk, cash flow, oral radiology, cost, investments

\section{Background and aims}

Radiological examinations are indispensable for the diagnosis and represent one of the main treatment planning methods used in all fields of medicine. Together with the benefits of X-rays, comes also one important issue represented by the fact that a certain amount of radiation is inevitably delivered to patients. Nowadays, the use of digital radiographs offers a potential dose reduction [1].

In dentistry, 2D X-rays (intraoral, panoramic and cephalometric $\mathrm{X}$ rays) and 3D Cone-Beam Computer Tomography (CBCT) are considered the starting point for endodontic, dental anomalies, malocclusions and maxillofacial traumatic emergencies. The increasing use of CBCT in dental medicine is a sensitive point considering the radiation doses involved, especially for pediatric patients. Thus, for some specific pathological conditions, the 3D imaging for children is justified. The use of CBCT plays an important role in the planning and treatment for impacted and supplementary teeth, orofacial cleft, dentoalveolar trauma and dental anomalies [2].

According to the UNSCEAR Report [1], dental examinations are the most frequent type of radiological procedures. The number of dental X-rays in Romania is increasing on a yearly basis. In 2008, the total number of dental examinations was 342.943 per year, while 
in 2014 it doubled, to 750.445 [3].

There is increased interest in the field of dental radiology in Romania [4].

Like dental practice, the oral radiological center is a private field. The current trend is that beside the private clinic, the dentist must also have a radiological center. Benefits do not only improve diagnosis, save time and increase the prestige of the clinic, but also increase the practice's profit. Investments in such devices are still complex, costly and even risky if they are not well documented and phased.

Considering the current interest and trend that dentists would install a radiology laboratory beside their current practice, this study investigates the issue of financial efficiency related to such investment in order to estimate the break-even-point of such investment, the financial efficiency to be expected and describe a financial model for assessing the financial risk of investment in an oral radiology laboratory that could be extended to others regions in Europe.

\section{Methods}

Our analysis took into consideration two alternatives: (A) investment in a simple radiology laboratory beside a current dental practice, consisting only of intraoral equipment, hereinafter referred to as "Intraoral Radiology" and (B) investment in a complex radiology center, comprising intraoral equipment and panoramicCBCT equipment, referred to as "Radiology Center".

The feasibility of an investment was analyzed by means of the return that may be obtained on that investment. The return on investment (ROI) was studied by means of the internal rate of return (IRR) and the net present value of the investment (NPV). NPV represents the present value of present and future cash flows. The investment is the sum to be paid for the achievement of the investment plan and represents a present negative cash flow. Future cash flows to be derived from operating such investment were discounted using a discount rate in order to reflect the money value over time. The sum of all these discounted cash flows represents the net present value of the investment. IRR represents the discount rate for which an investment is equal to the present value of the future expected benefits (cash flows) or the discount rate at which the present value of expected future benefits is equal to the investment (at break-even point). The investment was considered attractive if the IRR was higher than the discount rate.

The analysis of the financial feasibility considered the following steps, for each aforementioned alternative: (1) Estimation of the necessary investment; (2) Revenues forecast; (3) Expenses forecast; (4) Budget, working capital and capital investment forecast; (5) Cash flow forecast.

The analysis was made on a forecast period of 10 years, taking into account that this represents the minimum period of life of the acquired equipment. An average investment level was considered for the Intraoral Radiology, while in the case of the Radiology Centre, the analysis took into account a maximum investment level.

The Intraoral Radiology is operated by a dentist, hence the investment must be made in the form of a legal person paying corporate tax as all Romanian entities. In Romania, the corporate taxes amount to $16 \%$. In terms of location, the Intraoral Radiology is supposed to be a space ancillary to the dental practice.

In the present study the considered costs, fees and prices do not include VAT, and all the prices are estimated in euro (EUR).

\section{Revenues forecast}

The revenue forecast was estimated based on the following: the mean number of X-rays to be made and fees per X-ray. The mean number of X-rays to be made was estimated by months for the first year, when a monthly increase is considered. The monthly increase is based on the expectancy to increase the number of clients as the Radiology center is better known, achieving at the end of the year a number of $\mathrm{X}$-rays per day aligned to the current market expectations. For year two, the monthly number of X-rays is considered to remain constant, at the level of Month 12 from Year 1. Starting with Year 3, a small 1\% year by year increase was taken into account. The fees per $\mathrm{X}$-ray are based on current market fees. Starting with Year 2 , a small yearly $1 \%$ fee increase was taken into account.

Expenses forecast

Expenses forecast relied on the issues summarised in table I for the Oral Radiology center and table II for the Radiology Center. Expenses related to equipment, certifications, medical staff and location were considered.

Consumable costs per type of X-ray were estimated based on the numbers of X-rays to be made, for each type of radiological equipment and practice. Maintenance and certification costs include necessary costs with equipment accreditation and service and disinfection materials. For Year 1 these expenses, apart from the disinfection materials, were included in the initial investment.

Other expenses are estimated as $10 \%$ of total revenue. These include unplanned expenses, mainly administrative (cleaning, materials, telephone costs, promotion materials etc.).

Staff expenses are related to wages and wages costs and are based on the number of personnel, the average net wage of a radiologist and the expenses to be paid on wages. The staff includes only radiologists and the number increases to two subsequent to the number increase of daily X-rays. Medical training costs: are based on necessary certificates and are calculated based on the personnel number, certification costs and frequency.

The rent is estimated according to the area believed necessary (Intraoral Radiology: 20 sqm; Radiology Center: $40 \mathrm{sqm})$. Rent level is estimated based on current market quotations and location assumptions. Utilities (electricity, 
water, heating) cost is estimated based on the area and current market prices and are presented by a monthly average level.

Depreciation is estimated based on investment costs and depreciation duration taken into account ( 5 years for computers and 10 years for radiology equipment). Costs are supposed to increase with a $1 \%$ per year moderate increase, while utilities are estimated to grow by $5 \%$ yearly. forecast

Budget, working capital and capital investment

Budgets are estimated on the basis of expenses and revenues presented above. No financial revenues and expenses were considered.

\section{Cash flow forecast}

The cash flow for both alternatives was constructed based on the cash flow of equity model. Considering that no financing flows were taken into account, the cash flow includes cash flow from operations and cash flows from investment. The elements used are presented in tables XIII and XIV. Operational flows include net profit and working capital change. The net profit was taken from tables XI and XII. The working capital for each year was estimated based on a 30 days period rotation for inventory and payables. For receivables, a 1 to 5 days rotation period was considered (usually these services are paid upfront). Investment flows include depreciation as input and capital investment expenses (CAPEX) as expense. Depreciation was taken from tables XI and XII. Capex was estimated at a level of 10 to $25 \%$, beginning with the Third year, considering that only accidental replacements might be needed. In year Five, a new computer acquisition is scheduled.

Also, for estimating the net present value of the investments and the internal rate of return, a discount rate was estimated as measure of perceived risk, used to discount future expected benefits to a present value, thus reflecting the time value of money. The discount rate estimation was made based on the CAPM model.

\section{Results}

The estimated investment is presented in table I for Intraoral Radiology, respectively table II for Radiology Centre. Table 1 presents the minimum and maximum estimates and an average, which is the selected scenario. Table II presents only the minimum and maximum estimated investment, the selected scenario being the maximum investment.

Revenue forecasts for both practice types are presented monthly for the First year (table III and table V) and yearly for the entire forecast period (table IV and table VI). Estimated costs are presented monthly for the First year (table VII and table VIII) and yearly for the entire forecast period (table IX and table X).

Table I. Estimation of investment for Intraoral Radiology.

\begin{tabular}{|c|c|c|c|c|}
\hline \multicolumn{5}{|c|}{ Investment estimate - Intraoral Radiology } \\
\hline No & Investment item & \multicolumn{3}{|c|}{ Estimated cost } \\
\hline EUR & & Min & Max & Average \\
\hline \multirow[t]{7}{*}{1} & Equipment & & & \\
\hline & Intraoral equipment & 1,800 & 2,300 & 2,050 \\
\hline & Digital detector for intra-oral & 3,000 & 4,000 & 3,500 \\
\hline & Printer dry film & 5,000 & 6,000 & 5,500 \\
\hline & Radiosafety equipment & 320 & 370 & 345 \\
\hline & Computer & 700 & 1,200 & 950 \\
\hline & Total equipment & 10,820 & 13,870 & 12,345 \\
\hline \multirow[t]{4}{*}{2} & Location related costs & & & \\
\hline & Rent for the set-up duration & 400 & 600 & 250 \\
\hline & Radiolology cabinet fitting & 1,500 & 2,000 & 1,750 \\
\hline & Total location related costs & 1,900 & 2,600 & 2,000 \\
\hline \multirow[t]{5}{*}{3} & X-ray Laboratory Accreditation & & & \\
\hline & Radiological expertise & 200 & 300 & 250 \\
\hline & Public Health Authority & 100 & 100 & 100 \\
\hline & National Authority for Radioprotection & 600 & 600 & 600 \\
\hline & Total Accreditation costs & 900 & 1,000 & 950 \\
\hline \multirow[t]{4}{*}{4} & Staff training costs & & & \\
\hline & Health certificate & 20 & 30 & 25 \\
\hline & Individual dosimetry & 25 & 35 & 30 \\
\hline & Total staff training costs & 45 & 65 & 55 \\
\hline 5 & Marketing & 100 & 150 & 125 \\
\hline \multirow[t]{3}{*}{6} & Miscelleanous & 693 & 1,769 & 774 \\
\hline & Total & 14,458 & 19,454 & 16,249 \\
\hline & Total investment (rounded) & 14,500 & 19,500 & 16,200 \\
\hline
\end{tabular}


Table II. Estimation of investment for a Radiology Center.

\begin{tabular}{|c|c|c|c|}
\hline No & Investment item & \multicolumn{2}{|c|}{ Estimated cost } \\
\hline EUR & & Min & Max \\
\hline \multirow[t]{8}{*}{1} & Equipment & & \\
\hline & Intraoral equipment & 2,300 & 2,300 \\
\hline & Digital detector for intra-oral & 10,000 & 10,000 \\
\hline & $\mathrm{CBCT}+$ Panoramic & 120,000 & 200,000 \\
\hline & Printer dry film & 5,000 & 6,000 \\
\hline & Radiosafety equipment & 320 & 370 \\
\hline & Computer & 700 & 1,200 \\
\hline & Total equipment & 138,320 & 219,870 \\
\hline \multirow[t]{4}{*}{2} & Location related costs & & \\
\hline & Rent for the set-up duration & 600 & 1,000 \\
\hline & Radiolology cabinet fitting & 2,000 & 3,000 \\
\hline & Total location related costs & 2,600 & 4,000 \\
\hline \multirow[t]{5}{*}{3} & X-ray Laboratory Accreditation & & \\
\hline & Radiological expertise & 400 & 600 \\
\hline & Public Health Authority & 200 & 200 \\
\hline & National Authority for Radioprotection & 1,000 & 1,000 \\
\hline & Total Accreditation costs & 1,600 & 1,800 \\
\hline \multirow[t]{4}{*}{4} & Staff training costs & & \\
\hline & Health certificate & 20 & 30 \\
\hline & Individual dosimetry & 25 & 35 \\
\hline & Total staff training costs & 45 & 65 \\
\hline 5 & Marketing & 100 & 150 \\
\hline \multirow[t]{3}{*}{6} & Miscelleanous & 7,148 & 11,274 \\
\hline & Total investment & 149,813 & 237,159 \\
\hline & Grand Total & 150,000 & 237,000 \\
\hline
\end{tabular}

Table III. Revenues estimated monthly for the first year for Intraoral Radiology.

\begin{tabular}{|c|c|c|c|c|c|c|c|c|c|c|c|c|c|c|}
\hline \multirow[t]{2}{*}{ Description } & \multirow[t]{2}{*}{ MU } & \multicolumn{12}{|c|}{ Month } & \multirow{2}{*}{$\begin{array}{l}\text { Total } \\
\text { Year } 1\end{array}$} \\
\hline & & $\begin{array}{r}\text { Month } \\
1\end{array}$ & $\begin{array}{r}\text { Month } \\
2\end{array}$ & $\begin{array}{r}\text { Month } \\
3\end{array}$ & $\begin{array}{r}\text { Month } \\
4\end{array}$ & $\begin{array}{r}\text { Month } \\
5\end{array}$ & $\begin{array}{r}\text { Month } \\
6\end{array}$ & $\begin{array}{r}\text { Month } \\
7\end{array}$ & $\begin{array}{r}\text { Month } \\
8\end{array}$ & $\begin{array}{r}\text { Month } \\
9\end{array}$ & $\begin{array}{r}\text { Month } \\
10\end{array}$ & $\begin{array}{r}\text { Month } \\
11\end{array}$ & $\begin{array}{r}\text { Month } \\
12\end{array}$ & \\
\hline Number of radiographies & Pcs/month & 400 & 440 & 484 & 532 & 585 & 644 & 696 & 731 & 768 & 806 & 846 & 888 & 7,820 \\
\hline No increase factor & & & $0 \%$ & $10 \%$ & $10 \%$ & $10 \%$ & $10 \%$ & $10 \%$ & $8 \%$ & $5 \%$ & $5 \%$ & $5 \%$ & $5 \%$ & \\
\hline Unit free & EUR/Pcs & 3.25 & 3.25 & 3.25 & 3.25 & 3.25 & 3.25 & 3.25 & 3.25 & 3.25 & 3.25 & 3.25 & 3.25 & 3.25 \\
\hline $\begin{array}{l}\text { Revenue intraoral } \\
\text { radiography }\end{array}$ & EUR & 1,300 & 1,430 & 1,573 & 1,729 & 1,901 & 2,093 & 2,262 & 2,376 & 2,496 & 2,620 & 2,750 & 2,886 & 25,415 \\
\hline
\end{tabular}

Table IV. Revenues estimated yearly for Intraoral Radiology.

Estimated revenues for the first year - Intraoral Radiology

\begin{tabular}{|c|c|c|c|c|c|c|c|c|c|c|c|}
\hline \multirow[t]{2}{*}{ Description } & \multirow[t]{2}{*}{ MU } & \multicolumn{10}{|c|}{ Year } \\
\hline & & Year 1 & Year 2 & Year 3 & Year 4 & Year 5 & Year 6 & Year 7 & Year 8 & Year 9 & Year 10 \\
\hline Number of radiographies & Pcs/year & 7,820 & 10,656 & 10,763 & 10,871 & 10,980 & 11,090 & 11,201 & 11,313 & 11,426 & 11,540 \\
\hline No increase factor & & & & $1 \%$ & $1 \%$ & $1 \%$ & $1 \%$ & $1 \%$ & $1 \%$ & $1 \%$ & $1 \%$ \\
\hline Unit free & EUR/Pcs & 3.25 & 3.28 & 3.32 & 3.35 & 3.38 & 3.42 & 3.45 & 3.48 & 3.52 & 3.55 \\
\hline Fee increase factor & & & 0 & 0 & 0 & 0 & 0 & 0 & 0 & 0 & $1 \%$ \\
\hline Revenue intraoral radiography & EUR & 25,415 & 34,978 & 35,683 & 36,401 & 37,134 & $\mathbf{3 7 , 8 8 1}$ & 38,643 & 39,419 & 40,211 & 41,019 \\
\hline
\end{tabular}


Table V. Revenues estimated monthly for the first years for Radiology Center.

\begin{tabular}{|c|c|c|c|c|c|c|c|c|c|c|c|c|c|c|}
\hline \multicolumn{15}{|c|}{ Estimated revenues for the first year - Radiology Center } \\
\hline \multirow{2}{*}{ Description } & \multirow{2}{*}{ MU } & \multicolumn{11}{|c|}{ Month } & \multicolumn{2}{|r|}{ Total } \\
\hline & & $\begin{array}{r}\text { Month } \\
1\end{array}$ & $\begin{array}{r}\text { Month } \\
2\end{array}$ & $\begin{array}{r}\text { Month } \\
3\end{array}$ & $\begin{array}{r}\text { Month } \\
4\end{array}$ & $\begin{array}{r}\text { Month } \\
5\end{array}$ & $\begin{array}{r}\text { Month } \\
6\end{array}$ & $\begin{array}{r}\text { Month } \\
7\end{array}$ & $\begin{array}{r}\text { Month } \\
8\end{array}$ & $\begin{array}{r}\text { Month } \\
9\end{array}$ & $\begin{array}{r}\text { Month } \\
10\end{array}$ & $\begin{array}{r}\text { Month } \\
11\end{array}$ & $\begin{array}{r}\text { Month } \\
12\end{array}$ & Year 1 \\
\hline $\begin{array}{l}\text { Number of intraoral } \\
\text { radiographies }\end{array}$ & Pcs/month & 600 & 660 & 726 & 799 & 879 & 967 & 1,044 & 1,096 & 1,151 & 1,209 & 1,269 & 1,332 & 11,732 \\
\hline No increase factor & & & $10 \%$ & $10 \%$ & $10 \%$ & $10 \%$ & $10 \%$ & $10 \%$ & $8 \%$ & $5 \%$ & $5 \%$ & $5 \%$ & $5 \%$ & \\
\hline Unit free & EUR/Pcs & 3.20 & 3.20 & 3.20 & 3.20 & 3.20 & 3.20 & 3.20 & 3.20 & 3.20 & 3.20 & 3.20 & 3.20 & 3.20 \\
\hline $\begin{array}{l}\text { Revenue intraoral } \\
\text { radiography }\end{array}$ & EUR & 1,920 & 2,112 & 2,323 & 2,557 & 2,813 & 3,094 & 3,341 & 3,507 & 3,683 & 3.869 & 4,061 & 4,262 & 37,542 \\
\hline $\begin{array}{l}\text { Number of panoramic } \\
\text { radiographies }\end{array}$ & Pcs/month & 400 & 440 & 484 & 532 & 585 & 644 & 696 & 731 & 768 & 806 & 846 & 888 & 7,820 \\
\hline No increase factor & & & $10 \%$ & $10 \%$ & $10 \%$ & $10 \%$ & $10 \%$ & $10 \%$ & $8 \%$ & $5 \%$ & $5 \%$ & $5 \%$ & $5 \%$ & \\
\hline Unit free & EUR/Pcs & 7.00 & 7.00 & 7.00 & 7.00 & 7.00 & 7.00 & 7.00 & 7.00 & 7.00 & 7.00 & 7.00 & 7.00 & 7.00 \\
\hline $\begin{array}{l}\text { Revenue panoramic } \\
\text { radiography }\end{array}$ & EUR & 2,800 & 3,080 & 3,388 & 3,724 & 4,095 & 4,508 & 4,872 & 5,117 & 5,376 & 5,642 & 5,922 & 6,216 & 54,740 \\
\hline Number of CBCT & Pcs/month & 100 & 104 & 109 & 113 & 116 & 118 & 119 & 120 & 121 & 122 & 123 & 124 & 1,389 \\
\hline No increase factor & & & $4 \%$ & $5 \%$ & $4 \%$ & $3 \%$ & $2 \%$ & $1 \%$ & $1 \%$ & $1 \%$ & $1 \%$ & $1 \%$ & $1 \%$ & \\
\hline Unit free & EUR/Pcs & 45.00 & 45.00 & 45.00 & 45.00 & 45.00 & 45.00 & 45.00 & 45.00 & 45.00 & 45.00 & 45.00 & 45.00 & 45.00 \\
\hline Revenue CBCT & EUR & 4,500 & 4,680 & 4,905 & 5,085 & 5,220 & 5,310 & 5,355 & 5,400 & 5,445 & 5,490 & 5,535 & 5,580 & 62,505 \\
\hline Total revenue & EUR & 9,220 & 9,872 & 10,616 & 11,366 & 12,128 & 12,912 & 13,568 & 14,024 & 14,504 & 15,001 & 15,518 & 16,058 & 154,787 \\
\hline
\end{tabular}

Table VI. Revenues estimated yearly for Radiology Center.

\begin{tabular}{|c|c|c|c|c|c|c|c|c|c|c|c|}
\hline \multicolumn{12}{|c|}{ Estimated revenues - Radiology Center } \\
\hline \multirow[t]{2}{*}{ Description } & \multirow[t]{2}{*}{ MU } & \multicolumn{10}{|c|}{ Year } \\
\hline & & Year 1 & Year 2 & Year 3 & Year 4 & Year 5 & Year 6 & Year 7 & Year 8 & Year 9 & Year 10 \\
\hline $\begin{array}{l}\text { Number of intraoral } \\
\text { radiographies }\end{array}$ & Pcs/year & 11,732 & 15,984 & 16,144 & 16,305 & 16,468 & 16,633 & 16,799 & 16,967 & 17,137 & 17,308 \\
\hline No increase factor & & & & $1 \%$ & $1 \%$ & $1 \%$ & $1 \%$ & $1 \%$ & $1 \%$ & $1 \%$ & $1 \%$ \\
\hline Unit free & EUR/Pcs & 3.20 & 3.23 & 3.26 & 3.30 & 3.33 & 3.36 & 3.40 & 3.43 & 3.47 & 3.50 \\
\hline Fee increase factor & & $0 \%$ & $1 \%$ & $1 \%$ & $1 \%$ & $1 \%$ & $1 \%$ & $1 \%$ & $1 \%$ & $1 \%$ & $1 \%$ \\
\hline $\begin{array}{l}\text { Revenue intraoral } \\
\text { radiography }\end{array}$ & EUR & 37,542 & 51,660 & 52,699 & $\mathbf{5 3 , 7 5 7}$ & $\mathbf{5 4 , 8 3 7}$ & 55,941 & 57,064 & 58,211 & 59,382 & 60,574 \\
\hline $\begin{array}{l}\text { Number of panoramic } \\
\text { radiographies }\end{array}$ & Pcs/year & 7,820 & 10,656 & 10,763 & 10,871 & 10,980 & 11,090 & 11,201 & 11,313 & 11,426 & 11,540 \\
\hline No increase factor & & & & $1 \%$ & $1 \%$ & $1 \%$ & $1 \%$ & $1 \%$ & $1 \%$ & $1 \%$ & $1 \%$ \\
\hline Unit free & EUR/Pcs & 7.00 & 7.07 & 7.14 & 7.21 & 7.28 & 7.36 & 7.43 & 7.50 & 7.58 & 7.66 \\
\hline Fee increase factor & & $0 \%$ & $1 \%$ & $1 \%$ & $1 \%$ & $1 \%$ & $1 \%$ & $1 \%$ & $1 \%$ & $1 \%$ & $1 \%$ \\
\hline $\begin{array}{l}\text { Revenue panoramic } \\
\text { radiography }\end{array}$ & EUR & 54,740 & 75,338 & 76,855 & 78,403 & 79,981 & 81,590 & 83,231 & 84,903 & 86,609 & 88,348 \\
\hline Number of CBCT & Pcs/year & 1,389 & 1,488 & 1,503 & 1,518 & 1,533 & 1,548 & 1,563 & 1,579 & 1,595 & 1,611 \\
\hline No increase factor & & & & $1 \%$ & $1 \%$ & $1 \%$ & $1 \%$ & $1 \%$ & $1 \%$ & $1 \%$ & $1 \%$ \\
\hline Unit free & EUR/Pcs & 45.00 & 45.45 & 45.90 & 46.36 & 46.83 & 47.30 & 47.77 & 48.25 & 48.73 & 49.22 \\
\hline Fee increase factor & & $0 \%$ & $1 \%$ & $1 \%$ & $1 \%$ & $1 \%$ & $1 \%$ & $1 \%$ & $1 \%$ & $1 \%$ & $1 \%$ \\
\hline Revenue CBCT & EUR & 62,505 & 67,630 & 68,994 & 70,380 & 71,786 & 73,213 & 74,662 & 76,181 & 77,722 & 79,287 \\
\hline Total revenue & EUR & 154,787 & 194,628 & 198,549 & 202,540 & 206,604 & 210,744 & 214,957 & 219,295 & 223,713 & 228,209 \\
\hline
\end{tabular}


Table VII. Estimated costs presented monthly for the first year for Intraoral Radiology.

\begin{tabular}{|c|c|c|c|c|c|c|c|c|c|c|c|c|c|c|c|}
\hline \multirow[t]{3}{*}{ Description } & \multirow[t]{3}{*}{ MU } & \multirow[t]{3}{*}{$\begin{array}{l}\text { Unit } \\
\text { Cost }\end{array}$} & \multicolumn{12}{|c|}{ Month } & \multirow[t]{2}{*}{ Total } \\
\hline & & & Month & Month & Month & Month & Month & Month & Month & Month & Month & Month & Month & Month & \\
\hline & & & 1 & 2 & 3 & 4 & 5 & 6 & 7 & 8 & 9 & 10 & 11 & 12 & \\
\hline Number of radiographies & Pcs/month & & 400 & 440 & 484 & 532 & 585 & 644 & 696 & 731 & 768 & 806 & 846 & 888 & 7,820 \\
\hline Consumables & Eur & & 410 & 451 & 496 & 545 & 600 & 660 & 713 & 749 & 787 & 826 & 867 & 910 & 8,016 \\
\hline Consumable price & EUR/pcs & 1.00 & 400 & 440 & 484 & 532 & 585 & 644 & 696 & 731 & 768 & 806 & 846 & 888 & 7,820 \\
\hline Tonner & EUR/pcs & 0.025 & 10 & 11 & 12 & 13 & 15 & 16 & 17 & 18 & 19 & 20 & 21 & 22 & 196 \\
\hline $\begin{array}{l}\text { Maintenance and } \\
\text { certification costs }\end{array}$ & EUR & & - & - & - & - & - & - & - & - & - & - & - & - & 600 \\
\hline $\begin{array}{l}\text { Annual technical } \\
\text { certificate and service }\end{array}$ & EUR/year & 1000 & - & - & - & - & - & - & - & - & - & - & - & - & - \\
\hline $\begin{array}{l}\text { OTDM X-ray } \\
\text { equipments certificate }\end{array}$ & EUR/2 years & 500 & - & - & - & - & - & - & - & - & - & - & - & - & - \\
\hline $\begin{array}{l}\text { Radiosafety } \\
\text { equipments certificate }\end{array}$ & EUR/2 years & 350 & - & - & - & - & - & - & - & - & - & - & - & - & - \\
\hline Disinfection materials & EUR/year & 600 & 50 & 50 & 50 & 50 & 50 & 50 & 50 & 50 & 50 & 50 & 50 & 50 & 600 \\
\hline Other expenses & EUR & & 130 & 143 & 157 & 173 & 190 & 209 & 226 & 238 & 250 & 262 & 275 & 289 & 2,542 \\
\hline Staff expenses & EUR & 495 & 495 & 495 & 495 & 495 & 495 & 495 & 495 & 990 & 990 & 990 & 990 & 990 & 8,415 \\
\hline Number of persons & EUR & 1 & 1 & 1 & 1 & 1 & 1 & 1 & 1 & 2 & 2 & 2 & 2 & 2 & 2 \\
\hline $\begin{array}{l}\text { Wages and wages } \\
\text { expenses }\end{array}$ & EUR/person & 495 & 495 & 495 & 495 & 495 & 495 & 495 & 495 & 495 & 495 & 495 & 495 & 495 & 5,940 \\
\hline Medical training costs & EUR & & - & - & - & - & - & - & - & 45 & - & - & - & - & 45 \\
\hline Health certificate & EUR/5 years & 20 & - & - & - & - & - & - & - & 20 & - & - & - & - & 20 \\
\hline Individual dosimetry & EUR/5 years & 25 & - & - & - & - & - & - & - & 25 & - & - & - & - & 25 \\
\hline Rent & EUR/month & 100 & 100 & 100 & 100 & 100 & 100 & 100 & 100 & 100 & 100 & 100 & 100 & 100 & 1,200 \\
\hline Utilities & EUR/month & $\mathbf{5 0}$ & 50 & 50 & 50 & 50 & 50 & 50 & $\mathbf{5 0}$ & 50 & 50 & 50 & 50 & 50 & 600 \\
\hline Depreciation & EUR & & 111 & 111 & 111 & 111 & 111 & 111 & 111 & 111 & 111 & 111 & 111 & 111 & 1,330 \\
\hline Radiology equipment & EUR & 1139.5 & 95 & 95 & 95 & 95 & 95 & 95 & 95 & 95 & 95 & 95 & 95 & 95 & 1,140 \\
\hline Computer & EUR & 190.0 & 16 & 16 & 16 & 16 & 16 & 16 & 16 & 16 & 16 & 16 & 16 & 16 & 190 \\
\hline Total costs & EUR & & 1,166 & 1,207 & 1,252 & 1,301 & 1,355 & 1,416 & 1,469 & 2,045 & 2,038 & 2,077 & 2,118 & 2,1612 & 22,747 \\
\hline
\end{tabular}

Table VIII. Estimated costs presented monthly for the first year for Radiology Center.

\begin{tabular}{|c|c|c|c|c|c|c|c|c|c|c|c|c|c|c|c|}
\hline \multirow[t]{2}{*}{ Description } & \multirow[t]{2}{*}{ MU } & \multirow[t]{2}{*}{\begin{tabular}{|l|} 
Unit \\
Cost \\
\end{tabular}} & \multicolumn{12}{|c|}{ Month } & \multirow{2}{*}{$\begin{array}{l}\text { Total } \\
\text { Year } 1\end{array}$} \\
\hline & & & $\begin{array}{r}\text { Month } \\
1\end{array}$ & $\begin{array}{r}\text { Month } \\
2\end{array}$ & $\begin{array}{r}\text { Month } \\
3\end{array}$ & $\begin{array}{r}\text { Month } \\
4\end{array}$ & $\begin{array}{r}\text { Month } \\
5\end{array}$ & $\begin{array}{r}\text { Month } \\
6\end{array}$ & $\begin{array}{r}\text { Month } \\
7\end{array}$ & $\begin{array}{r}\text { Month } \\
8\end{array}$ & $\begin{array}{r}\text { Month } \\
9\end{array}$ & $\begin{array}{r}\text { Month } \\
10\end{array}$ & $\begin{array}{r}\text { Month } \\
11\end{array}$ & $\begin{array}{r}\text { Month } \\
12\end{array}$ & \\
\hline $\begin{array}{l}\text { Total number of } \\
\text { radiographies; of which }\end{array}$ & Pcs/month & & 1,100 & 1,204 & 1,319 & 1,444 & 1,580 & 1,729 & 1,859 & 1,947 & 2,040 & 2,137 & 2,238 & 2,344 & 20,941 \\
\hline Intraoral & Pcs/month & & 600 & 660 & 726 & 799 & 879 & 967 & 1,044 & 1,096 & 1,151 & 1,209 & 1,269 & 1,332 & 11,732 \\
\hline Panoramic & Pcs/month & & 400 & 440 & 484 & 532 & 585 & 644 & 696 & 731 & 768 & 806 & 846 & 888 & 7,820 \\
\hline$C B C T$ & Pcs/month & & 100 & 104 & 109 & 113 & 116 & 118 & 119 & 120 & 121 & 122 & 123 & 124 & 1,389 \\
\hline Consumables & EUR & & 1,050 & 1,154 & 1,268 & 1,393 & 1,530 & 1,681 & 1,813 & 1,903 & 1,997 & 2,096 & 2,199 & 2,307 & 20,388 \\
\hline Consumable price & EUR/pes & 1.000 & 1.000 & 1,100 & 1,210 & 1,331 & 1,464 & 1,611 & 1,740 & 1,827 & 1,919 & 2,015 & 2,115 & 2,220 & 19,552 \\
\hline Tonner & EUR/pcs & 0.025 & 25 & 28 & 30 & 33 & 37 & 40 & 44 & 46 & 48 & 50 & 53 & 56 & 489 \\
\hline $\mathrm{CD}$ price (for $\mathrm{CBCT}$ ) & EUR/pcs & 0.250 & 25 & 26 & 27 & 28 & 29 & 30 & 30 & 30 & 30 & 31 & 31 & 31 & 347 \\
\hline $\begin{array}{l}\text { Maintenance and } \\
\text { certification costs }\end{array}$ & EUR & & 100 & 100 & 100 & 100 & 100 & 100 & 100 & 100 & 100 & 100 & 100 & 2,100 & 3,200 \\
\hline $\begin{array}{l}\text { Annual technical } \\
\text { certificate and service }\end{array}$ & EUR/year & 2,000 & - & - & - & - & - & - & - & - & - & - & - & 2,000 & 2,000 \\
\hline $\begin{array}{l}\text { OTDM X-ray } \\
\text { equipments certificate }\end{array}$ & EUR/2 years & 1,000 & - & - & - & - & - & - & - & - & - & - & - & - & - \\
\hline $\begin{array}{l}\text { Radiosafety } \\
\text { equipments certificate }\end{array}$ & EUR/2 years & 350 & - & - & - & - & - & - & - & - & - & - & - & - & - \\
\hline Disinfection materials & EUR/year & 1,200 & 100 & 100 & 100 & 100 & 100 & 100 & 100 & 100 & 100 & 100 & 100 & 100 & 1,200 \\
\hline Other expenses & EUR & & 922 & 987 & 1,062 & 1,137 & 1,213 & 1,291 & 1,357 & 1,402 & 1,450 & 1,500 & 1,552 & 1,606 & 15,479 \\
\hline Staff expenses & EUR & & 1,890 & 1,890 & 1,890 & 1,890 & 1,890 & 1,890 & 1,890 & 1,890 & 1,890 & 1,890 & 1,890 & 1,890 & 22,680 \\
\hline Number of persons & & 3 & 3 & 3 & 3 & 3 & 3 & 3 & 3 & 3 & 3 & 3 & 3 & 3 & 3 \\
\hline $\begin{array}{l}\text { Wages and wages } \\
\text { expenses }\end{array}$ & EUR/person & 630 & 630 & 630 & 630 & 630 & 630 & 630 & 630 & 630 & 630 & 630 & 630 & 630 & 7,560 \\
\hline Medical training costs & EUR & & - & - & - & - & - & - & - & - & - & - & - & - & - \\
\hline Health & EUR/5 years & 20 & - & - & - & - & - & - & - & - & - & - & - & - & - \\
\hline Individual dosimetry & EUR/5 years & 25 & - & - & - & - & - & - & - & - & - & - & - & - & - \\
\hline Rent & EUR/month & 500 & 500 & 500 & 500 & 500 & 500 & 500 & 500 & 500 & 500 & 500 & 500 & 500 & 6,000 \\
\hline Utilities & EUR/month & 70 & 70 & 70 & 70 & 70 & 70 & 70 & 70 & 70 & 70 & 70 & 70 & 70 & 840 \\
\hline Depreciation & EUR & & 1,842 & 1,842 & 1,842 & 1,842 & 1,842 & 1,842 & 1,842 & 1,842 & 1,842 & 1,842 & 1,842 & 1,842 & 22,107 \\
\hline Radiology equipment & EUR & 21,867 & 1,822 & 1,822 & 1,822 & 1,822 & 1,822 & 1,822 & 1,822 & 1,822 & 1,822 & 1,822 & 1,822 & 1,822 & 21,867 \\
\hline Computer & EUR & 240 & 20 & 20 & 20 & 20 & 20 & 20 & 20 & 20 & 20 & 20 & 20 & 20 & 240 \\
\hline Total costs & EUR & & 6,374 & 6,543 & 6,731 & 6,931 & 7,145 & 7,374 & 7,572 & 7,707 & 7,850 & 7,998 & 8,153 & 10,315 & 90,694 \\
\hline
\end{tabular}


Table IX. Estimated costs presented yearly for Intraoral Radiology.

\begin{tabular}{|c|c|c|c|c|c|c|c|c|c|c|c|}
\hline \multicolumn{12}{|c|}{ Estimated costs - Intraoral Radiology } \\
\hline \multirow[t]{2}{*}{ Description } & \multirow[t]{2}{*}{ MU } & \multicolumn{10}{|c|}{ Year } \\
\hline & & Year 1 & Year 2 & Year 3 & Year 4 & Year 5 & Year 6 & Year 7 & Year 8 & Year 9 & Year 10 \\
\hline Number of radiographies & Pcs/month & 7,820 & 10,656 & 10,763 & 10,871 & 10,980 & 11,090 & 11,201 & 11,313 & 11,426 & 11,540 \\
\hline Consumables & EUR & 8,016 & 11,032 & 11,251 & 11,475 & 11,703 & 11,936 & 12,173 & 12,415 & 12,661 & 12,913 \\
\hline Consumable price & $E U R / p c s$ & 7,820 & 10,763 & 10,979 & 11,200 & 11,426 & 11,656 & 11,890 & 12,129 & 12,373 & 12,621 \\
\hline Tonner & $E U R / p c s$ & 196 & 269 & 272 & 274 & 277 & 280 & 283 & 286 & 289 & 291 \\
\hline $\begin{array}{l}\text { Maintenance and } \\
\text { certification costs }\end{array}$ & EUR & 600 & 1,859 & 1,020 & 1,936 & 1,051 & 2,034 & 1,173 & 2,254 & 1,361 & 2,579 \\
\hline $\begin{array}{l}\text { Annual technical certificate } \\
\text { and service }\end{array}$ & EUR/year & - & 1,000 & 1,020 & 1,051 & 1,051 & 1,105 & 1,173 & 1,257 & 1,361 & 1,489 \\
\hline $\begin{array}{l}\text { OTDM } X \text {-ray equipments } \\
\text { certificate }\end{array}$ & EUR/2 years & - & 505 & - & 520 & - & 547 & - & 586 & - & 641 \\
\hline $\begin{array}{l}\text { Radiosafety equipments } \\
\text { certificate }\end{array}$ & EUR/2 years & - & 354 & - & 364 & - & 383 & - & 410 & - & 449 \\
\hline Disinfection materials & EUR/year & 600 & 606 & 618 & 637 & 663 & 697 & 739 & 793 & 858 & 939 \\
\hline Other expenses & EUR & 2,542 & 3,498 & 3,568 & 3,640 & 3,713 & 3,788 & 3,864 & 3,942 & 4,021 & 4,102 \\
\hline Staff expenses & EUR & 8,415 & 11,999 & 12,119 & 12,240 & 12,362 & 12,486 & 12,611 & 12,737 & 12,864 & 12,993 \\
\hline Number of persons & EUR & 2 & 2 & 2 & 2 & 2 & 2 & 2 & 2 & 2 & 2 \\
\hline Wages and wages expenses & EUR/person & 5,940 & 5,999 & 6,059 & 6,120 & 6,181 & 6,243 & 6,305 & 6,368 & 6,432 & 6,496 \\
\hline Medical training costs & EUR & 45 & - & - & - & 47 & 47 & - & - & 48 & - \\
\hline Health certificate & EUR/5 years & 20 & - & - & - & 21 & 21 & - & - & 21 & - \\
\hline Individual dosimetry & EUR $/ 5$ years & 25 & - & - & - & 26 & 26 & - & - & 27 & - \\
\hline Rent & EUR/month & 1,200 & 1,212 & 1,224 & 1,236 & 1,249 & 1,261 & 1,274 & 1,287 & 1,299 & 1,312 \\
\hline Utilities & EUR/month & 600 & 630 & 662 & 695 & 729 & 766 & 804 & 844 & 886 & 931 \\
\hline Depreciation & EUR & 1,330 & 1,330 & 1,330 & 1,330 & 1,330 & 1,330 & 1,330 & 1,330 & 1,330 & 1,330 \\
\hline Radiology equipment & $E U R$ & 1,140 & 1,140 & 1,140 & 1,140 & 1,140 & 1,140 & 1,140 & 1,140 & 1,140 & 1,140 \\
\hline Computer & $E U R$ & 190 & 190 & 190 & 190 & 190 & 190 & 190 & 190 & 190 & 190 \\
\hline Total costs & EUR & 22,747 & 32,164 & 31,792 & 33,188 & 32,847 & 34,344 & 33,967 & 35,601 & 35,330 & 37,098 \\
\hline
\end{tabular}

Table X. Estimated costs presented yearly for Radiology Center.

\begin{tabular}{|c|c|c|c|c|c|c|c|c|c|c|c|}
\hline \multicolumn{12}{|c|}{ Estimated costs -Radiology Center } \\
\hline \multirow[t]{2}{*}{ Description } & \multirow[t]{2}{*}{ MU } & \multicolumn{10}{|c|}{ Year } \\
\hline & & Year 1 & Year 2 & Year 3 & Year 4 & Year 5 & Year 6 & Year 7 & Year 8 & Year 9 & Year 10 \\
\hline $\begin{array}{l}\text { Total number of radiographies; } \\
\text { of which }\end{array}$ & Pcs/month & 20,941 & 28,128 & 28,410 & 28,694 & 28,981 & 29,271 & 29,563 & 29,859 & 30,158 & 30,459 \\
\hline Intraoral & Pcs/month & 11,732 & 15,984 & 16,144 & 16,305 & 16,468 & 16,633 & 16,799 & 16,967 & 17,137 & 17,308 \\
\hline Panoramic & Pcs/month & 7,820 & 10,656 & 10,763 & 10,871 & 10,980 & 11,090 & 11,201 & 11,313 & 11,426 & 11,540 \\
\hline$C B C T$ & Pcs/month & 1,389 & 1,488 & 1,503 & 1,518 & 1,533 & 1,548 & 1,563 & 1,579 & 1,595 & 1,611 \\
\hline Consumables & $E \boldsymbol{E}$ & 20,388 & 27,955 & 28,235 & 28,517 & 28,803 & 29,091 & 29,382 & 29,676 & 29,973 & 30,272 \\
\hline Consumable price & $E U R / p c s$ & 19,552 & 26,906 & 27,176 & 27,448 & 27,722 & 28,000 & 28,280 & 28,563 & 28,849 & 29,136 \\
\hline Tonner & $E U R / p c s$ & 489 & 673 & 679 & 686 & 693 & 700 & 707 & 714 & 721 & 728 \\
\hline$C D$ price (for $C B C T$ ) & $E U R / p c s$ & 347 & 376 & 380 & 383 & 387 & 391 & 395 & 399 & 403 & 407 \\
\hline $\begin{array}{l}\text { Maintenance and certification } \\
\text { costs }\end{array}$ & EUR & $\mathbf{3 , 2 0 0}$ & 3,384 & 2,040 & 3,465 & 2,081 & 3,578 & 2,123 & 3,727 & 2,166 & 3,919 \\
\hline $\begin{array}{l}\text { Annual technical certificate } \\
\text { and service }\end{array}$ & EUR/year & 2,000 & 2,020 & 2,040 & 2,061 & 2,081 & 2,102 & 2,123 & 2,144 & 2,166 & 2,187 \\
\hline $\begin{array}{l}\text { OTDM X-ray equipments } \\
\text { certificate }\end{array}$ & EUR/2 years & - & 1,010 & - & 1,041 & - & 1,094 & - & 1,173 & - & 1,282 \\
\hline $\begin{array}{l}\text { Radiosafety equipments } \\
\text { certificate }\end{array}$ & EUR/2 years & - & 354 & - & 364 & - & 383 & - & 410 & - & 449 \\
\hline Disinfection materials & EUR/year & 1,200 & 1,212 & 1,236 & 1,274 & 1,326 & 1,393 & 1,479 & 1,586 & 1,717 & 1,878 \\
\hline Other expenses & EUR & 15,479 & 19,463 & 19,855 & 20,254 & 20,660 & 21,074 & 21,496 & 21,929 & 22,371 & 22,821 \\
\hline Staff expenses & EUR & 22,680 & 22,907 & 23,136 & 23,367 & 23,601 & 23,837 & 24,075 & 24,316 & 24,559 & 24,805 \\
\hline Number of persons & - & 3 & 3 & 3 & 3 & 3 & 3 & 3 & 3 & 3 & 3 \\
\hline Wages and wages expenses & EUR/person & 7,560 & 7,636 & 7,712 & 7,789 & 7,867 & 7,946 & 8,025 & 8,105 & 8,186 & 8,268 \\
\hline Medical training costs & $E U R$ & - & - & - & - & 142 & - & - & - & - & 149 \\
\hline Health certificate & $E U R / 5$ years & - & - & - & - & 21 & - & - & - & - & 22 \\
\hline Individual dosimetry & $E U R / 5$ years & - & - & - & - & 26 & - & - & - & - & 28 \\
\hline Rent & EUR/month & 6,000 & 6,060 & 6,121 & 6,182 & 6,244 & 6,306 & 6,369 & 6,433 & 6,497 & 6,562 \\
\hline Utilities & EUR/month & 840 & 882 & 926 & 972 & 1,021 & 1,072 & 1,126 & 1,182 & 1,241 & 1,303 \\
\hline Depreciation & EUR & 22,107 & 22,107 & 22,107 & 22,107 & 22,107 & 22,107 & 22,107 & 22,107 & 22,107 & 22,107 \\
\hline Radiology equipment & EUR & 21,867 & 21,867 & 21,867 & 21,867 & 21,867 & 21,867 & 21,867 & 21,867 & 21,867 & 21,867 \\
\hline Computer & EUR & 240 & 240 & 240 & 240 & 240 & 240 & 240 & 240 & 240 & 240 \\
\hline Total costs & EUR & 90,694 & 103,969 & 103,656 & 106,139 & 105,984 & 108,459 & 108,156 & 110,956 & 110,631 & 113,815 \\
\hline
\end{tabular}


Table XI. Yearly estimated budgets for Intraoral Radiology.

\begin{tabular}{|c|c|c|c|c|c|c|c|c|c|c|}
\hline \multicolumn{11}{|c|}{ Estimated Budget - Intraoral Radiology } \\
\hline \multirow{2}{*}{$\begin{array}{l}\text { EUR } \\
\text { Description }\end{array}$} & \multicolumn{10}{|c|}{ Year } \\
\hline & Year 1 & Year 2 & Year 3 & Year 4 & Year 5 & Year 6 & Year 7 & Year 8 & Year 9 & Year 10 \\
\hline Total Revenues & 25,415 & 34,978 & 35,683 & 36,401 & 37,134 & 37,881 & 38,643 & 39,419 & 40,211 & 41,019 \\
\hline Total costs & $(21,417)$ & $(\mathbf{3 0 , 8 3 5 )}$ & $(30,462)$ & $(31,858)$ & $(31,517)$ & $(33,014)$ & $(32,638)$ & $(34,271)$ & $(33,999)$ & $(35,768)$ \\
\hline Consumables & $(8,016)$ & $(11,032)$ & $(11,251)$ & $(11,475)$ & $(11,703)$ & $(11,936)$ & $(12,173)$ & $(12,415)$ & $(12,661)$ & $(12,913)$ \\
\hline $\begin{array}{l}\text { Maintenance and } \\
\text { certification costs }\end{array}$ & $(600)$ & $(2,465)$ & $(1,638)$ & $(2,572)$ & $(1,714)$ & $(2,731)$ & $(1,912)$ & $(3,047)$ & $(2,220)$ & $(3,518)$ \\
\hline Other expenses & $(2,542)$ & $(3,498)$ & $(3,568)$ & $(3,640)$ & $(3,713)$ & $(3,788)$ & $(3,864)$ & $(3,942)$ & $(4,021)$ & $(4,102)$ \\
\hline Staff expenses & $(8,415)$ & $(11,999)$ & $(12,119)$ & $(12,240)$ & $(12,362)$ & $(12,486)$ & $(12,611)$ & $(12,737)$ & $(12,864)$ & $(12,993)$ \\
\hline Medical training costs & (45) & - & - & - & (47) & (47) & - & - & (47) & - \\
\hline Rent & $(1,200)$ & $(1,212)$ & $(1,224)$ & $(1,236)$ & $(1,249)$ & $(1,261)$ & $(1,274)$ & $(1,287)$ & $(1,299)$ & $(1,312)$ \\
\hline Utilities & $(600)$ & (630) & $(662)$ & $(695)$ & $(729)$ & $(766)$ & $(804)$ & $(844)$ & $(886)$ & (931) \\
\hline EBITDA & 3,998 & 4,144 & 5,221 & 4,543 & 5,616 & 4,867 & 6,005 & 5,148 & 6,212 & 5,250 \\
\hline Depreciation & $(1,330)$ & $(1,330)$ & $(1,330)$ & $(1,330)$ & $(1,330)$ & $(1,330)$ & $(1,330)$ & $(1,330)$ & $(1,330)$ & $(1,330)$ \\
\hline EBIT & 2,669 & 2,814 & 3,891 & 3,213 & 4,287 & 3,537 & 4,675 & 3,819 & 4,883 & 3,921 \\
\hline Corporate tax & (427) & $(450)$ & (623) & (514) & (686) & (566) & $(3,587)$ & (611) & (781) & (627) \\
\hline Net profit & 2,242 & 2,364 & 3,269 & 2,699 & 3,601 & 2,971 & 1,088 & 3,208 & 4,101 & 3,293 \\
\hline
\end{tabular}

Table XII. Yearly estimated budgets for Radiology Center.

\begin{tabular}{|c|c|c|c|c|c|c|c|c|c|c|}
\hline \multicolumn{11}{|c|}{ Estimated Budget-Radiology Center } \\
\hline EUR & \multicolumn{10}{|c|}{ Year } \\
\hline Description & Year 1 & Year 2 & Year 3 & Year 4 & Year 5 & Year 6 & Year 7 & Year 8 & Year 9 & Year 10 \\
\hline Total Revenues & 154,787 & 194,628 & 198,549 & 202,540 & 206,604 & 210,744 & 214,957 & 219,295 & 223,713 & 228,209 \\
\hline Total costs & $(68,587)$ & $(81,862)$ & $(81,549)$ & $(84,032)$ & $(83,877)$ & $(86,352)$ & $(86,049)$ & $(88,849)$ & $(88,524)$ & $(91,708)$ \\
\hline Consumable price & $(20,388)$ & $(27,955)$ & $(28,235)$ & $(28,517)$ & $(28,803)$ & $(29,091)$ & $(29,382)$ & $(29,676)$ & $(29,973)$ & $(30,272)$ \\
\hline $\begin{array}{l}\text { Maintenance and } \\
\text { certification costs }\end{array}$ & $(3,200)$ & $(4,596)$ & $(3,277)$ & $(4,739)$ & $(3,407)$ & $(4,972)$ & $(3,602)$ & $(5,313)$ & $(3,883)$ & $(5,796)$ \\
\hline Other expenses & $(15,479)$ & $(19,463)$ & $(19,855)$ & $(20,254)$ & $(20,660)$ & $(21,074)$ & $(21,496)$ & $(21,929)$ & $(22,371)$ & $(22,821)$ \\
\hline Staff expenses & $(22,680)$ & $(22,907)$ & $(23,136)$ & $(23,367)$ & $(23,601)$ & $(23,837)$ & $(24,075)$ & $(24,316)$ & $(24,559)$ & $(24,805)$ \\
\hline Medical training costs & - & - & - & - & $(142)$ & - & - & - & - & (149) \\
\hline Rent & $(6,000)$ & $(6,060)$ & $(6,121)$ & $(6,182)$ & $(6,244)$ & $(6,306)$ & $(6,369)$ & $(6,433)$ & $(6,497)$ & $(6,562)$ \\
\hline Utilities & $(840)$ & $(882)$ & (926) & (972) & $(1,021)$ & $(1,072)$ & $(1,126)$ & $(1,182)$ & $(1,241)$ & $(1,303)$ \\
\hline EBITDA & 86,201 & 112,766 & 117,000 & 118,508 & 122,727 & 124,392 & 128,907 & 130,446 & 135,189 & 136,501 \\
\hline Depreciation & $(22,107)$ & $(22,107)$ & $(22,107)$ & $(22,107)$ & $(22,107)$ & $(22,107)$ & $(22,107)$ & $(22,107)$ & $(22,107)$ & $(22,107)$ \\
\hline EBIT & 64,094 & 90,659 & 94,893 & 96,401 & 100,620 & 102,285 & 106,800 & 108,339 & 113,082 & 114,394 \\
\hline Corporate tax & $(10,255)$ & $(14,505)$ & $(15,183)$ & $(15,424)$ & $(16,099)$ & $(16,366)$ & $(17,088)$ & $(17,334)$ & $(18,093)$ & $(18,303)$ \\
\hline Net profit & 53,839 & 76,154 & 79,710 & 80,977 & 84,521 & 85,919 & 89,712 & 91,005 & 94,989 & 96,091 \\
\hline
\end{tabular}

Table XIII. Cash flows for Intraoral Radiology.

\begin{tabular}{|c|c|c|c|c|c|c|c|c|c|c|c|}
\hline \multicolumn{12}{|c|}{ Estimated Cash Flows - Intraoral Radiology } \\
\hline \multicolumn{2}{|c|}{ EUR } & \multicolumn{10}{|c|}{ Year } \\
\hline Description & & Year 1 & Year 2 & Year 3 & Year 4 & Year 5 & Year 6 & Year 7 & Year 8 & Year 9 & Year 10 \\
\hline Net profit & & 2,242 & 2,364 & 3,269 & 2,699 & 3,601 & 2,971 & 1,088 & 3,208 & 4,101 & 3,293 \\
\hline Depreciation & & 1,330 & 1,330 & 1,330 & 1,330 & 1,330 & 1,330 & 1,330 & 1,330 & 1,330 & 1,330 \\
\hline WACC change & & (71) & (124) & (103) & (6) & (6) & (6) & (6) & (6) & (7) & (7) \\
\hline CAPEX & & - & - & (130) & (130) & (130) & (680) & (330) & (330) & (330) & (330) \\
\hline Total cash flow & $(16,200)$ & 3,500 & 3,570 & 4,365 & 3,893 & 4,794 & 3,614 & 2,082 & 4,201 & 5,094 & 4,286 \\
\hline Discount rate & $8.25 \%$ & & & & & & & & & & \\
\hline Investment & 16,200 & & & & & & & & & & \\
\hline NPV & 8,949 & & & & & & & & & & \\
\hline IRR & $20.0 \%$ & & & & & & & & & & \\
\hline
\end{tabular}


Table XIV. Cash flows for Radiology Center.

\begin{tabular}{|c|c|c|c|c|c|c|c|c|c|c|c|}
\hline \multicolumn{12}{|c|}{ Estimated Cash Flows -Radiology Center } \\
\hline \multirow{2}{*}{$\begin{array}{l}\text { EUR } \\
\text { Description }\end{array}$} & & \multicolumn{10}{|c|}{ Year } \\
\hline & & Year 1 & Year 2 & Year 3 & Year 4 & Year 5 & Year 6 & Year 7 & Year 8 & Year 9 & Year 10 \\
\hline Net profit & & 53,839 & 76,154 & 79,710 & 80,977 & 84,521 & 85,919 & 89,712 & 91,005 & 94,989 & 96,091 \\
\hline Depreciation & & 22,107 & 22,107 & 22,107 & 22,107 & 22,107 & 22,107 & 22,107 & 22,107 & 22,107 & 22,107 \\
\hline WACC change & & $(860)$ & (762) & (584) & (607) & (56) & (57) & (59) & $(60)$ & (61) & (62) \\
\hline CAPEX & & - & - & $(2,210)$ & $(2,210)$ & $(2,210)$ & $(3,410)$ & $(2,210)$ & $(2,210)$ & $(2,210)$ & $(22,107)$ \\
\hline Total cash flow & $(237,000)$ & 75,086 & 97,499 & 99,023 & 100,267 & 104,361 & 104,559 & 109,551 & 110,842 & 114,825 & 96,028 \\
\hline Discount rate & $9.25 \%$ & & & & & & & & & & \\
\hline Investment & 237,000 & & & & & & & & & & \\
\hline NPV & 360,014 & & & & & & & & & & \\
\hline IRR & $38.0 \%$ & & & & & & & & & & \\
\hline
\end{tabular}

The yearly budgets for the Intraoral Radiology Laboratory and the Radiology center are presented in table $\mathrm{XI}$ and table XII, respectively.

Tables XIII and XIV present the cash flows, discount rate, NPV and IRR for each alternative considered. In both alternatives, the net present values (NPV) and the internal rate of return (IRR) show that the investment is feasible and that, in a 10 year term, there is a positive substantial return.

\section{Discussion}

Nowadays, a number of intraoral and extra-oral imaging modalities are available to assist the radiological examination in dental medicine, one important reason for dentists to also have a Radiology Center. Commonly used two-dimensional (2D) modalities, which include bitewing, periapical and panoramic X-ray are suitable because they are easily acquired, inexpensive and provide high-resolution images, however none without limitations: overlapping anatomical structures, difficulty in standardization, underestimating size and bone defect [5].

The 3D imaging technique, such as the Cone Beam Computed Tomography (CBCT) images, started to be used more often by the dentists as it carries highly informative value. The use of CBCT in clinical practice offers a number of potential advantages over conventional tomography and 2D techniques, including easier image acquisition, high image accuracy, reduced artefacts and lower effective radiation doses [6].

Therefore, for dental practice, we took into account two investment types, the first being an Intraoral Radiological center and, owing to the benefits provided by the CBCT imaging, the second investment type proposed in the current study was a Radiology Center.

Regarding the estimated cost of the two investment types, they vary substantially. The estimated cost for the Radiology Center is about ten times higher than for the Intraoral Center. This difference arises from the price of the CBCT machine, which is six time higher than the price of the intraoral equipment. Another important difference is represented by the fact that in the case of the Radiology Center, beside the CBCT equipment it also requires intraoral and panoramic radiological equipment [7].

The risk model used in the current study, the Capital Asset Pricing Model (CAPM) offers the possibility to compare different versions of placement in the financial markets. On the other hand, it justifies the estimation made on scientific basis of the expected future value of profits generated by a financial instrument. Thus, CAMP model approximated with accuracy the differences between the two models of investment proposed in this paper [7].

The current study anticipated that the cost difference between $\mathrm{CBCT}$ and conventional imaging methods may vary both within and also among different clinical conditions. Hence, a certain clinical condition of the patient may require a varying number of $\mathrm{CBCT}$ volumes or the use of different sizes of the field of view.

Higher volumes may represent a wider range of conditions for which CBCT is used, larger populations, different thresholds for referral, different catchment populations or a combination of these factors [8].

Thus, in many clinical conditions as dental anomalies, implantology, orthognathic surgery, endodontic assessments, CBCT examination is required even if it is more expensive, almost two times higher than the price of an intraoral X-ray [9].

This study examines a financial model to estimate financial risk, which could be extended at European level. Financial risk appears due to the instability and loss in the financial market caused by movements in stock prices and currencies, and is inherent in any investment, its proper manager being key in running a successful business [10]. The risk model used for the two investment types presented here, namely the CAMP model is widely used to estimate financial risk. 
CAPM is designed to establish a link between the risk of an asset and its expected return and it is frequently used due to the simplicity of the method and also because it offers the possibility of easy comparisons of investment alternatives [11].

Regarding the estimation of financial risk, there are several factors that should be taken into consideration: increased volatility of financial markets; developments in technology; increased number and model of radiology equipment, government regularization, liquidity and cash flow [12].

Starting from the idea that financial risk influenced directly and indirectly the profits of a business nowadays, certain companies from Europe introduced risk committees, whose main task is to identify, monitor and manage the company's risks [12].

The Radiology Center and the Intraoral Radiology presented in the current study are managed by the dentist. The major focus of the dentist should be to reduce harm and injury to both the patients and the radiological technician and to improve outcomes from the Radiology Center [13].

Hence, certain risks may be directly controlled, while others depend on factors unrelated to the dentist's managerial skills. The best thing a manager can do is to try to anticipate potential controllable risks, assess the potential impact on the business and be prepared with a second plan to react [14].

As regards the Intraoral Radiology investment, it is noticeably a more sensitive case. The return, if the maximum investment level is chosen, is much smaller than in the average case and twice as small in NPV terms. Also, investment is recovered in a period of 5 to 6 years.

Therefore, an investment in a Radiology Center is much more profitable and the return is much higher. The investment may be recovered in a 3 to 4 years' time and the IRR is much higher. With an internal rate of return much higher, the Radiology Center provides a much better chance of strong development. Nevertheless, we have to take into account that the magnitude of the initial investment is quite large. This would usually involve a quota of borrowed capital, meaning interest payment, hence a more reduced IRR.

The current study has some limitations. Those limits concern the fact that the CAMP model consists in determining a linear relationship between profitability and the financial market risk assumed by an investor, in this case the dentist. In reality, there are some external factors that influence the financial model estimation, such as interest rate changes, inflation, recession and changes in the market demand [12].

\section{Conclusions}

This study reveals an important input for economic evaluation in comparing costs and profits for two types of investments, Intraoral Radiology and Radiology Center. According to our findings, the Radiological center represents a more profitable investment due to a higher economic rate of return, even if the initial estimated budget is substantially larger than the budget for Intraoral Radiology.

\section{References}

1. Okano T, Sur J. Radiation dose and protection in dentistry. Japanese Dental Science Review. 2010;46:112-121.

2. Oenning AC, Jacobs R, Pauwels R, Stratis A, Hedesiu M, Salmon B, et al. Cone-beam $\mathrm{CT}$ in paediatric dentistry: DIMITRA project position statement. Pediatr Radiol. 2018;48:308-316.

3. European Commission. Radiation Protection 180. Medical radiation exposure for the European population. Luxembourg: Office for Official Publications of the European Communities 2014. Available from :https://ec.europa.eu/ energy/en/content/rp-180-medical-radiation-exposureeuropean-population-part-1-part-2

4. Sorop I, Mossang D, Iacob MR, Dadulescu E, Iacob O. Update of diagnostic medical and dental x-ray exposures in Romania. J Radiol Prot. 2008;28:563-571.

5. Mol A. Imaging methods in periodontology. Periodontol 2000. 2004;34:34-48.

6. Scarfe WC, Farman AG, Sukovic P. Clinical applications of cone-beam computed tomography in dental practice. J Can Dent Assoc. 2006;72:75-80.

7. Anghel MG, Paschia L. Using the CAMP model to estimate the profitability of a financial instrument portfolio. Annales Universitatis Apulensis Series Oeconomica. 2013;15:541551.

8. Christell H, Birch S, Hedesiu M, Horner K, Ivanauskaité $\mathrm{D}$, Nackaerts $\mathrm{O}$, et al. Variation in cost of cone beam CT examinations among healthcare systems. Dentomaxillofac Radiol. 2012;41:571-577.

9. Christell H, Birch S, Horner K, Rohlin M, Lindh C; SEDENTEXCT consortium. A framework for costing diagnostic methods in oral health care: an application comparing a new imaging technology with the conventional approach for maxillary canines with eruption disturbances. Community Dent Oral Epidemiol. 2012;40:351-361.

10. Verma E. Financial risk and its types. Available from: https:// www.simplilearn.com/financial-risk-and-types-rar131-article

11. Kenton W. Capital asset pricing model (CAMP). Available from: https://www.investopedia.com/terms/c/capm.asp

12. Vasilescu L. Financial Risk Management - Influence Factors and New Trends. 42 ${ }^{\text {th }}$ ed. Craiova: Annals of University of Craiova - Economic Sciences Series; 2014: pp. 69-75.

13. Craciun H, Mankad K, Lynch J. Risk management in radiology departments. World J Radiol. 2015;7:134-138.

14. Birch S, Gafni A. Economics and the evaluation of health care programmes: generalisability of methods and implications for generalisability of results. Health Policy. 2003;64:207219. 